\title{
Alterations in enhancer of zeste homolog 2, matrix metalloproteinase-2 and tissue inhibitor of metalloproteinase-2 expression are associated with $e x$ vivo and in vitro bone metastasis in renal cell carcinoma
}

\author{
JIANG WANG ${ }^{*}$, YE REN* ${ }^{*}$ XIN GUO, HAO CHENG, YAPING YE, JUN QI, CAIHONG YANG and HONGBO YOU \\ Department of Orthopedics, Tongji Hospital, Tongji Medical College, Huazhong University of Science and Technology, \\ Wuhan, Hubei 430030, P.R. China
}

Received March 11, 2014; Accepted December 9, 2014

DOI: $10.3892 / \mathrm{mmr} .2015 .3164$

\begin{abstract}
Renal cell carcinoma (RCC) has a high potential for bone metastasis; however, the molecular mechanisms underlying this metastasis have remained to be elucidated. The present study aimed to explore the expression levels of enhancer of zeste homolog 2 (EZH2), matrix metalloproteinase-2 (MMP2) and tissue inhibitor of metalloproteinase-2 (TIMP2) as determinants of RCC-associated bone metastasis. Their expression was evaluated in a newly generated RCC cell subline that has a high potential for bone metastasis, in tissue specimens from metastasized bone tissues from patients with RCC and in RCC tissues without metastasis. A total of 25 RCC tissue specimens without metastasis and 13 RCC tissue specimens with bone metastasis were acquired for immunohistochemical analysis of EZH2, MMP2 and TIMP2 protein expression. The expression levels of EZH2, MMP2 and TIMP2 mRNA and protein were analyzed in the ACHN and ACHN-BO5 cell lines using western blot and reverse transcription polymerase chain reaction (PCR) analyses. Methylation-specific PCR was also used to analyze TIMP2 promoter methylation. EZH2 and MMP2 proteins were found to be expressed at higher levels in tissues from patients where RCC had metastasized to the bone as compared with those in RCC patients without metastasis, whereas there was no significant difference in the expression of TIMP2
\end{abstract}

Correspondence to: Dr Hongbo You, Department of Orthopedics, Tongji Hospital, Tongji Medical College, Huazhong University of Science and Technology, 1095 Jie Fang Avenue, Wuhan, Hubei 430030, P.R. China

E-mail: hbyou003@gmail.com

*Contributed equally

Key words: renal cell carcinoma, enhancer of zeste homolog 2, matrix metalloproteinase-2, tissue inhibitor of metalloproteinase-2, tissue inhibitor of metalloproteinase- 2 promoter methylation, bone metastasis protein between the two tissues. Furthermore, the expression of EZH2 protein was correlated with MMP2 expression, but there was no significant correlation between the expression of $\mathrm{EZH} 2$ and TIMP2 proteins. The in vitro results using cell lines confirmed the ex vivo findings, indicating that the expression levels of $\mathrm{EZH} 2$ and MMP2 protein and mRNA were higher in ACHN-BO5 cells than those in ACHN cells. By contrast, TIMP2 protein and mRNA expression levels were lower in ACHN-BO5 cells than those in the parental ACHN cells. The TIMP2 promoter was highly methylated in ACHN-BO5 cells compared with that in ACHN cells. Upregulation of EZH2, MMP2 and TIMP2 expression was correlated with metastasis of RCC to bone tissues ex vivo and in vitro. Further studies are required in order to elucidate the mechanism underlying the altered expression of these genes.

\section{Introduction}

Renal cell carcinoma ( $\mathrm{RCC}$ ), which has been suggested to originate from the renal tubules and collecting tube epithelial cells, accounts for $85 \%$ of malignant kidney neoplasms and $\sim 2 \%$ of all human malignancies $(1,2)$. RCC is a pathologically heterogeneous disease, which can be classified into clear, papillary, granular, spindle and mixed cell subtypes based on certain cytoplasmic features (3). RCC morbidity increases by $2 \%$ annually and mortality has reached $\sim 100,000$ cases/year worldwide (4). Approximately $30 \%$ of patients with RCC develop metastatic disease, most frequently in the lungs, bones or brain (5). A clinical study confirmed that osteolysis represented $30 \%$ of the total metastatic disease cases associated with RCC (6). The incidence rate of bone tissue metastasis was higher in autopsy data from patients with RCC (5). The prognosis of RCC patients is influenced by a variety of factors, including tumor size, invasion, metastasis, histological type and nuclear grade (7); the five-year survival rate of patients with RCC was $90 \%$ for stage I, $51 \%$ for stage II, $22 \%$ for stage III and $4.6 \%$ for stage IV (8). Therefore, the elucidation of the key factors and molecular mechanisms underlying the metastasis of RCC to bone is required. A previous study by our group established an ACHN cell subline (ACHN-BO5) with high potential of bone 
metastasis compared with that of ACHN cells in animals in order to aid the elucidation of the underlying molecular mechanisms (9). In this subline, the pathological karyokinesis was significantly increased, which indicated that the malignant phenotype of the ACHN subline was higher than that of the parental ACHN cells. Following five passages of in vitro culture, the subline was named 'ACHN-BO5'. Subsequently, the gene alterations responsible for the high potential of bone metastasis were investigated using a complementary DNA (cDNA) microarray analysis to compare ACHN-BO5 cells with the parental ACHN cells. Alterations in the expression of enhancer of zeste homolog 2 (EZH2) and matrix metalloproteinase-2 (MMP2) were detected in ACHN-BO5 cells. EZH2 is involved in maintaining the transcriptional repressive state in cells and mutation of EZH2 causes Weaver syndrome (10), a congenital disorder associated with rapid growth beginning in the prenatal period, resulting in a characteristic facial appearance and certain skeletal features (11). In addition, studies have demonstrated that altered EZH2 expression promotes human cancer development (12-16). Proteins of the MMP family degrade or break down the extracellular matrix during normal physiological processes, including embryonic development and tissue remodeling, but also have a significant role in tumor metastasis (17). By contrast, tissue inhibitor of metalloproteinase-2 (TIMP2) is a natural matrix metalloproteinase inhibitor. Therefore, these proteins may have a role in mediating the metastasis of RCC to bone. In the present study, the expression of EZH2, MMP2 and TIMP2 were evaluated in RCC tissue specimens with or without bone metastasis and in ACHN-BO5 and ACHN cells to elucidate the correlation between their expression and metastasis in bone. Methylation-specific PCR analysis of TIMP2 promoter methylation was also performed. The results of the present study may aid the elucidation of the mechanisms underlying RCC metastasis and provide potential therapeutic targets for the prevention or treatment of RCC metastasis.

\section{Materials and methods}

Cell lines and culture. The human renal carcinoma cell line ACHN was obtained from the China Center of Type Culture Collection (Wuhan, China). The ACHN-B05 cell line was a subline of ACHN with high potential of bone metastasis, established in a previous study by our group (9). These cell lines were cultured in Dulbecco's modified Eagle's medium (DMEM; Invitrogen Life Technologies, Carlsbad, CA, USA) supplemented with $10 \%$ fetal bovine serum (FBS; Invitrogen Life Technologies) at $37^{\circ} \mathrm{C}$ in a humidified incubator with $5 \% \mathrm{CO}_{2}$.

Tissue specimens and immunohistochemistry. Primary renal cancer biopsy specimens $(n=25)$ and biopsies of renal cancer that had metastasized to bone tissues $(n=13)$ were obtained from The Tongji Hospital affiliated with Huazhong University of Science and Technology (Wuhan, China) between March 2010 and April 2013. The normal control renal biopsy specimens were obtained from adjacent normal tissues. The present study was approved by the ethics committee of Tongji Hospital affiliated with Huazhong University of Science and Technology. Written informed consent was obtained from all patients or their family. All tissues were fixed in $4 \%$ paraformaldehyde solution (Boster Biological Technology, Ltd., Wuhan, China) for $20 \mathrm{~min}$ at room temperature and embedded into paraffin using a routine tissue process (18). Tissue sections (4- $\mu \mathrm{M}$ thick) were prepared from the paraffin blocks and mounted onto glass slides. For immunohistochemical analysis, tissue sections were deparaffinized and rehydrated in water. The sections were heated in a pressure cooker $\left(121^{\circ} \mathrm{C}, 4 \mathrm{~min}\right)$ in a citric acid buffer (Boster Biological Technology, Ltd.) for antigen retrieval and then incubated with $3 \% \mathrm{H}_{2} \mathrm{O}_{2}$ /phosphate-buffered saline (PBS; Boster Biological Technology, Ltd.) at room temperature for $30 \mathrm{~min}$ to block potential peroxidase activity. Following incubation with $20 \%$ normal serum (Boster Biological Technology, Ltd.) for $30 \mathrm{~min}$, the sections were further incubated with primary antibodies: Mouse monoclonal immunoglobulin $\mathrm{G}_{1}\left(\mathrm{IgG}_{1}\right)$ anti-MMP2 antibody at a dilution of 1:800 (sc-13594; Santa Cruz Biotechnology, Inc., Dallas, TX, USA), goat polyclonal IgG anti-TIMP2 antibody at a dilution of 1:600 (sc-6835; Santa Cruz Biotechnology Inc.) or an goat polyclonal IgG anti-EZH2 antibody at a dilution of 1:100 (E6906; Sigma-Aldrich, St. Louis, MO, USA) overnight at $4^{\circ} \mathrm{C}$. The following day, the sections were washed three times with PBS and subsequently incubated with the secondary antibodies (anti-mouse IgG and anti-goat IgG horseradish peroxidase; Boster Biological Technology, Ltd.) for $1 \mathrm{~h}$ at $37^{\circ} \mathrm{C}$. A color reaction was performed using 3,3'-diaminobenzidine (Boster Biological Technology, Ltd.) as the chromogen. Diluted Sav-HRP conjugates were applied to the sections on the slides and incubated in a humidified chamber at room temperature for $30 \mathrm{~min}$ (protected from the light). Slides were washed with PBS twice, for 5 min each. DAB substrate solution (freshly made just before use: $0.05 \%$ DAB $-0.015 \% \mathrm{H}_{2} \mathrm{O}_{2}$ in PBS) was applied to the sections on the slides to reveal the color of antibody staining. The stained tissue sections were independently reviewed and scored under an Olympus CKX31 inverted microscope (Olympus Corp., Tokyo, Japan) by two investigators. The statistical results were analyzed using Sigmaplot 11.0 software (Systat Software, Inc., Chicago, IL, USA).

DNA extraction and methylation-specific PCR (MSP). Genomic DNA was extracted from ACHN and ACHN-BO5 cell lines using a Genomic DNA extraction kit (Boehringer, Mannheim, Germany) according to the manufacturer's instructions. DNA concentration, purity and integrity were measured using a spectrophotometer (Gilford 250; Gilford Instrument Laboratories, Inc., Oberlin, OH, USA) and gel electrophoresis. For MSP, genomic DNA samples $(2 \mu \mathrm{g})$ were denatured with sodium hydroxide chemically modified with sodium bisulfite (Boster Biological Technology, Ltd.). An MSP primer for the amplification of the TIMP2 promoter was designed using Methprime software (http://www.urogene.org/methprimer/index1.html). The methylation primers of the TIMP2 promoter were as follows: Forward, 5'-TTTTATTGTAGGAAAGGTCGA-3' and reverse, 5'-GAAATCATAAAACAACGCGTA-3', which amplified a 159-bp PCR product. The demethylation primers of the TIMP2 promoter used were: Forward, 5'-GAAGGAATATTTTATTGTAGGAAAGGTT-3' and reverse, 5'-TATAACACAAAATCATAAAACAACACATA-3', which amplified a 176-bp PCR product. PCR amplification 
Table I. Primers for PCR amplification of gene expression.

\begin{tabular}{llr}
\hline Gene & \multicolumn{1}{c}{ Sequences } & Size of PCR products (bp) \\
\hline EZH2 & 5'-GTGGAGAGATTATTTCTCAAGATG-3' & 289 \\
MMP2 & 5'-CCGACATACTTCAGGGCATCAGCC-3' & 207 \\
TIMP2 & 5'-GAGAACCAAAGTCTGAAGAG-3' & \\
& 5'-GGAGTGAGAATGCTGATTAG-3' & 89 \\
GAPDH & 5'-CCTCGGCCTTTC CTGCAAT-3' & 226
\end{tabular}

PCR, polymerase chain reaction; EZH2, enhancer of zeste homolog 2; MMP2, matrix metalloproteinase-2; TIMP2, tissue inhibitor of metalloproteinase-2.

occurred in a final volume of $25 \mu 1$ containing: $2.5 \mu 1 \mathrm{PCR}$ buffer, $2 \mu \mathrm{l} \mathrm{MgCl}_{2}, 2.5 \mu \mathrm{l}$ deoxynucleotide triphosphate mixture, $1 \mu \mathrm{l}$ of each primer, $5 \mu \mathrm{l}$ modified DNA template, $10.85 \mu \mathrm{l}$ sterilized deionized water and $0.15 \mu \mathrm{l}$ Taq enzyme (Takara Bio, Inc., Otsu, Japan). The PCR conditions were set at a pre-denaturation temperature of $94^{\circ} \mathrm{C}$ for $5 \mathrm{~min}$ followed by 45 cycles of $94^{\circ} \mathrm{C}$ for $30 \mathrm{sec}, 60^{\circ} \mathrm{C}$ (methylated) or $55^{\circ} \mathrm{C}$ (demethylated) for $45 \mathrm{sec}, 75^{\circ} \mathrm{C}$ for $40 \mathrm{sec}$ and a final extension at $73^{\circ} \mathrm{C}$ for $5 \mathrm{~min}$. Genomic DNA chemically modified with sss1 methylation enzyme (New England Biolabs, Shanghai, China) and bisulfite salts (Sigma-Aldrich) was used as a positive control. Deionized water was used as a negative control. PCR products were isolated using agarose gel electrophoresis and imaged under an ultraviolet lamp (XX15B, Spectronics Corp., Westbury, NY, USA).

RNA isolation and semi-quantitative RT-PCR. The expression levels of EZH2, MMP2 and TIMP2 mRNA were evaluated using RT-PCR analysis. Briefly, total cellular RNA was isolated from cells using TRIzol reagent (Invitrogen Life Technologies) and reverse-transcribed to cDNA using the First Strand cDNA Synthesis kit (Rever Tra Ace- $\alpha$; ToYoBo Co., Ltd., Osaka, Japan) according to the manufacturer's instructions. The primer sequences of genes and fragment sizes are indicated in Table I. The PCR conditions were as follows: Pre-denaturation at $94^{\circ} \mathrm{C}$ for $5 \mathrm{~min}$ and 32 cycles of $94^{\circ} \mathrm{C}$ for $45 \mathrm{sec} ; 52.5^{\circ} \mathrm{C}, 56^{\circ} \mathrm{C}$ and $54^{\circ} \mathrm{C}$ as the annealing temperature for $1 \mathrm{~min}, 72^{\circ} \mathrm{C}$ for $90 \mathrm{sec}$ and a final extension at $72^{\circ} \mathrm{C}$ for $10 \mathrm{~min}$. The PCR product was subsequently sequenced and identified by gel electrophoresis.

Protein extraction and western blot analysis. Cells were collected and lysed with a pre-cooled cell lysis buffer (Boster Biological Technology, Ltd.) containing $100 \mathrm{mM}$ Tris- $\mathrm{HCl}$, $500 \mathrm{mM}$ EDTA, $20 \mathrm{mM} \mathrm{NaCl}$, and 10\% SDS. The protein concentration was determined using a Bicinchoninic Acid Assay kit (Sigma-Aldrich, Irvine, Scotland). Briefly, an equal amount of protein sample was separated by SDS-PAGE and transferred onto a polyvinylidene fluoride membrane (A-FIT Biosciences, Beijing, China). For western blot analysis, the membranes were incubated in $5 \%$ skimmed milk/PBS at room temperature for $1 \mathrm{~h}$ and then further incubated with
anti-MMP2 antibody at a dilution of 1:800, anti-TIMP2 antibody at a dilution of 1:600 or anti-EZH2 antibody at a dilution of $1: 100$ at $4^{\circ} \mathrm{C}$ overnight. The following day, the membranes were washed three times with PBS-Tween-20 and incubated with a horseradish peroxidase-conjugated secondary antibody at a dilution of 1:7,500 at room temperature for $2 \mathrm{~h}$. Immunoreactive proteins were visualized using enhanced chemiluminescence (Pierce Biotechnology, Inc., Rockford, IL, USA) according to the manufacturer's instructions and exposed to X-ray films (Kodak, Rochester, NY, USA). The expression levels of these proteins were normalized to an internal control, GAPDH.

Statistical analysis. Values are presented as the mean \pm standard deviation of three independent experiments. All statistical analyses were performed using SPSS 11.0 software (SPSS, Inc., Chicago, IL, USA). The expression levels of EZH2, MMP2, TIMP2 mRNA and protein in ACHN and ACHN-B05 cells were compared using one-way analysis of variance. Differences in TIMP2 promoter methylation were analyzed using a $\chi^{2}$ test. A Pearson's correlation test was used to analyze the associations between different groups. $\mathrm{P}<0.05$ was considered to indicate a statistically significant difference between values.

\section{Results}

Differential expression of EZH2, MMP2 and TIMP2 proteins in tissues of patients with RCC as well as ACHN and $A C H N-B 05$ cells. In the present study, the expression of EZH2, MMP2 and TIMP2 proteins in tissues from patients with RCC with and without bone metastasis were evaluated by immunohistochemical analysis. The expression of EZH2 protein was higher in tissues from patients with RCC that had metastasized to the bone than in tissues of patients with RCC without metastasis ( $\mathrm{P}=0.031$; Fig. 1A-D). Analogously, expression levels of MMP2 protein were also higher in tissues from patients where $\mathrm{RCC}$ had metastatized than those in patients with RCC without metastasis $(\mathrm{P}=0.047$; Fig. 2A-D). By contrast, there were no significant differences in the expression of TIMP2 protein between the tissue types ( $\mathrm{P}=0.2932$; Fig. 3A-D). Furthermore, the expression of EZH2 and MMP2 proteins were found to be 

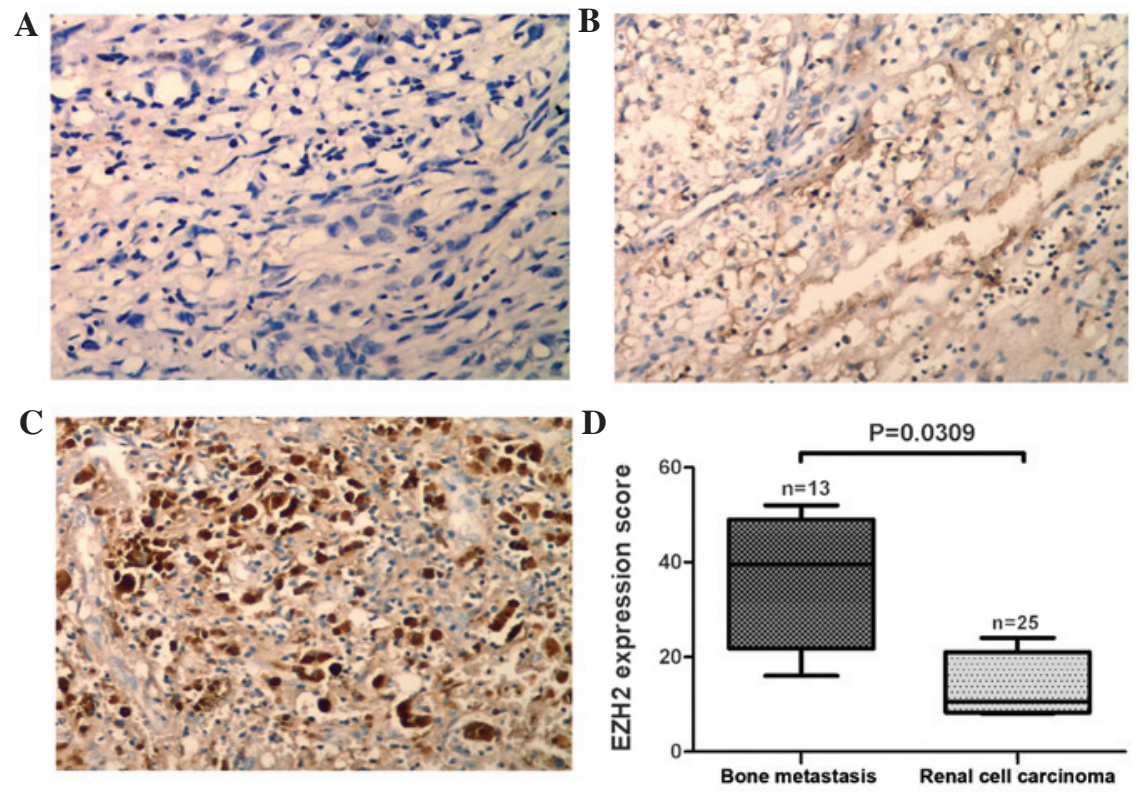

Figure 1. Immunohistochemical detection of EZH2 proteins in tissue sections of renal cancer patients with or without bone metastasis (magnification, $\mathrm{x} 400$ ). (A) EZH2-control; (B) EZH2 expression in RCC tissue; (C) EZH2 expression in tissue of patients with RCC with bone metastasis; (D) quantification of immunohistochemical analysis, comparing EZH2 expression score in tissues of RCC with and without bone metastasis. Images are representative of three independent experiments. EZH2, enhancer of zeste homolog 2; RCC, renal cell carcinoma.
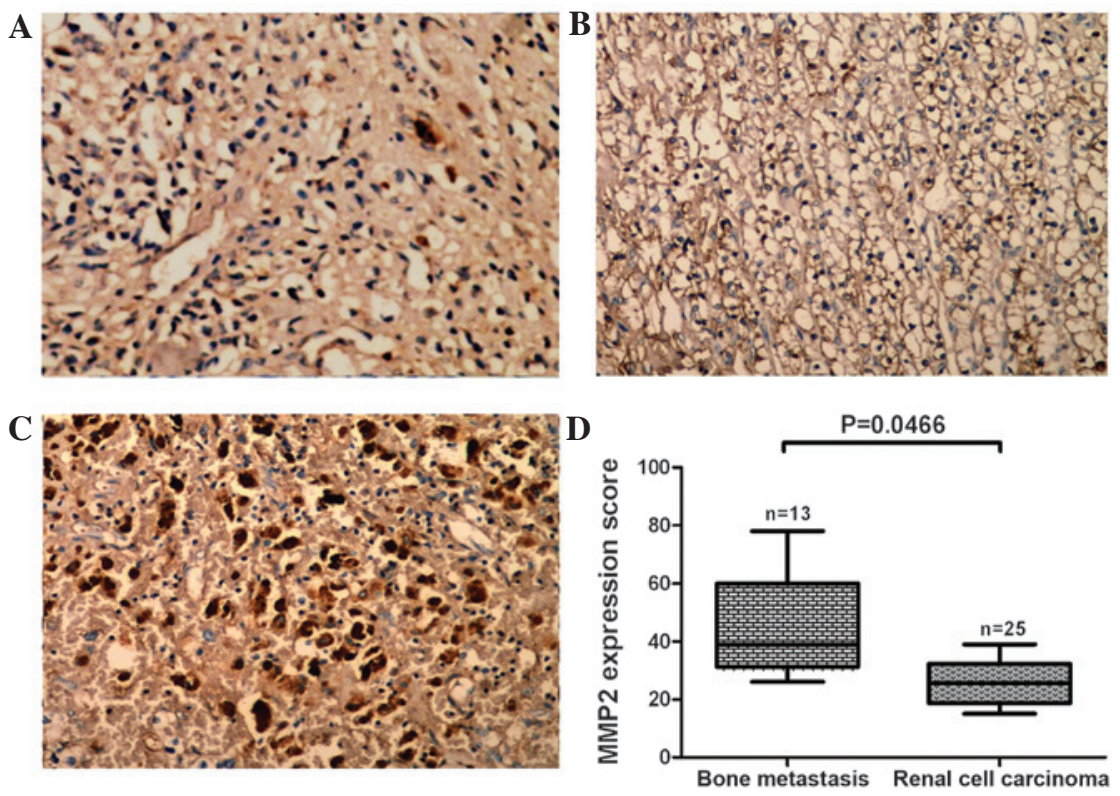

Figure 2. Immunohistochemical detection of MMP2 protein expression in tissue sections of renal cancer patients with or without bone metastasis (magnification, x400). (A) MMP2 control; (B) MMP2 expression in RCC tissue; (C) MMP2 expression an RCC tissue specimen with bone metastasis; (D) quantification of immunohistochemical analysis, comparing MMP2 expression levels in RCC tissues with and without bone metastasis. Images are representative of three independent experiments. MMP2, matrix metalloproteinase-2; RCC, renal cell carcinoma.

correlated $(\mathrm{r}=0.6652 ; \mathrm{P}=0.0131 ;$ Fig. $4 \mathrm{~A})$, whereas there was no significant correlation between EZH2 and TIMP2 protein expression ( $\mathrm{r}=-0.5484 ; \mathrm{P}=0.0523$; Fig. 4B).

Western blot analysis was used to investigate the expression levels of EZH2 protein in ACHN and ACHN-BO5 cells. EZH2 protein expression levels were demonstrated to be higher in ACHN-BO5 cells, a sub-line of ACHN with a higher potential for metastasis to the bone, than those in the parental ACHN cells $(\mathrm{P}<0.05)$, suggesting that $\mathrm{EZH} 2$ protein may be involved in mediating the metastasis of RCC to bone (Fig. 5A). In addition, the expression levels of MMP2 protein were higher in ACHN-BO5 cells than those in the parental ACHN cells $(\mathrm{P}<0.05)$. By contrast, TIMP2 protein expression levels were lower in ACHN-BO5 cells than those in the parental ACHN cells ( $\mathrm{P}<0.05$; Fig. 5B).

EZH2, MMP2 and TIMP2 mRNA expression levels differ between ACHN and ACHN-BO5 cells. RT-PCR analysis was 
A

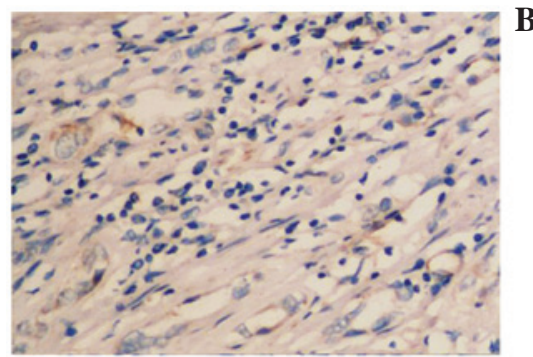

C

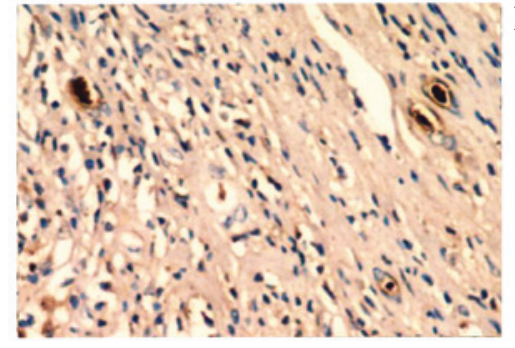

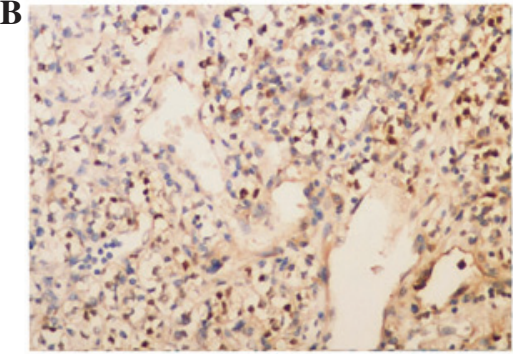

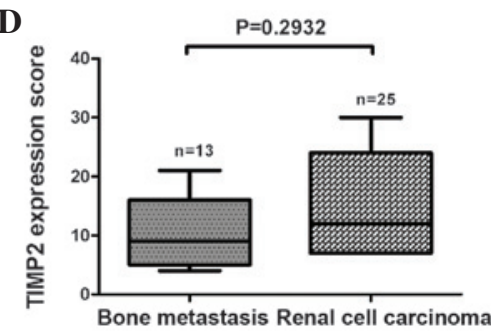

Figure 3. Immunohistochemical analysis of TIMP2 protein expression in tissue sections of renal cancer patients with or without bone metastasis (magnification, x400). (A) TIMP2 control; (B) TIMP2 expression in RCC tissue; (C) TIMP2 expression in RCC with bone metastasis; (D) quantification of immunohistochemical analysis results, comparing TIMP2 expression score in tissues of RCC with and without bone metastasis. Images are representative of three independent experiments. TIMP2, tissue inhibitor of metalloproteinase-2; RCC, renal cell carcinoma.
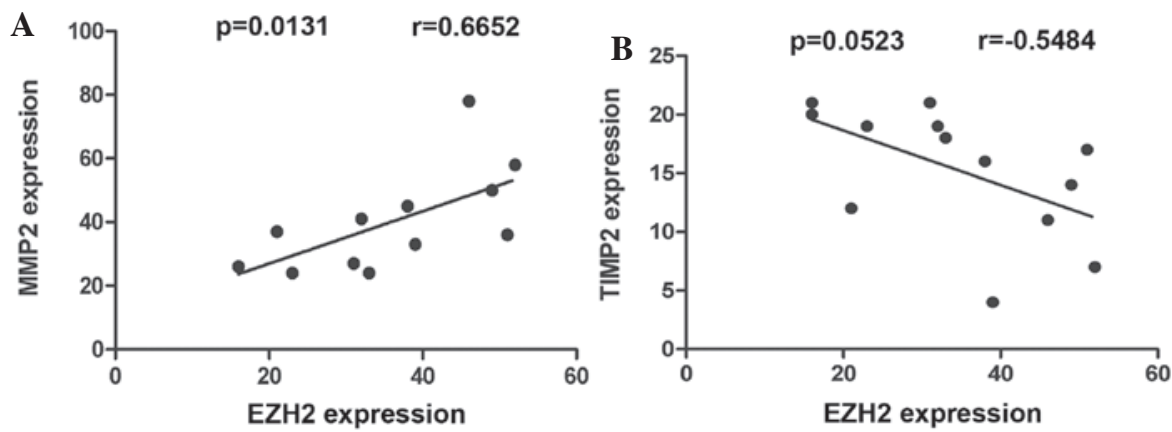

Figure 4. Evaluation of the correlation between EZH2 expression and MMP2 and TIMP2 protein in tissue sections of patients with RCC with or without bone metastasis using Spearman's test. (A) Correlation between MMP2 and EZH2 expression; (B) correlation between TIMP2 and EZH2 expression. EZH2, enhancer of zeste homolog 2; MMP2, matrix metalloproteinase-2; TIMP2, tissue inhibitor of metalloproteinase-2; RCC, renal cell carcinoma.

A

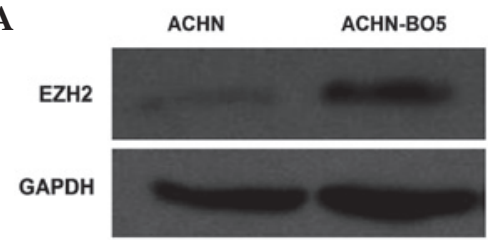

B

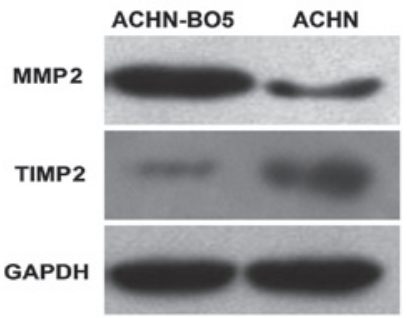

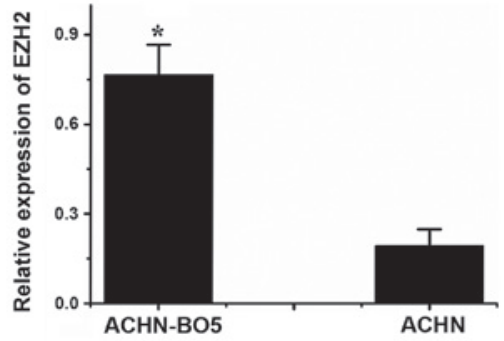

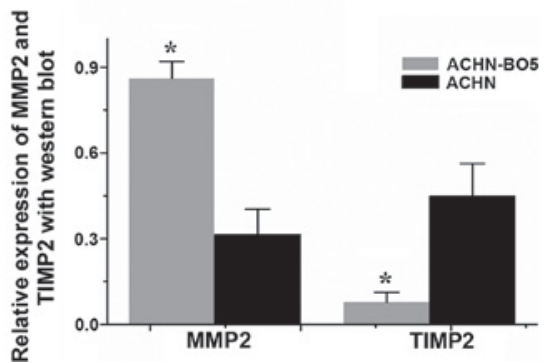

Figure 5. Western blot analysis of the expression levels of EZH2, MMP2 and TIMP2 proteins in ACHN and ACHN-BO5 cells. The graphs represent the quantified data from the western blot analyses. (A) Protein expression levels of EZH2; (B) protein expression levels of MMP2 and TIMP2. Values are presented as the mean \pm standard deviation $(\mathrm{n}=3) .{ }^{*} \mathrm{P}<0.05$ vs. ACHN cells. EZH2, enhancer of zeste homolog 2; MMP2, matrix metalloproteinase-2; TIMP2, tissue inhibitor of metalloproteinase-2. 


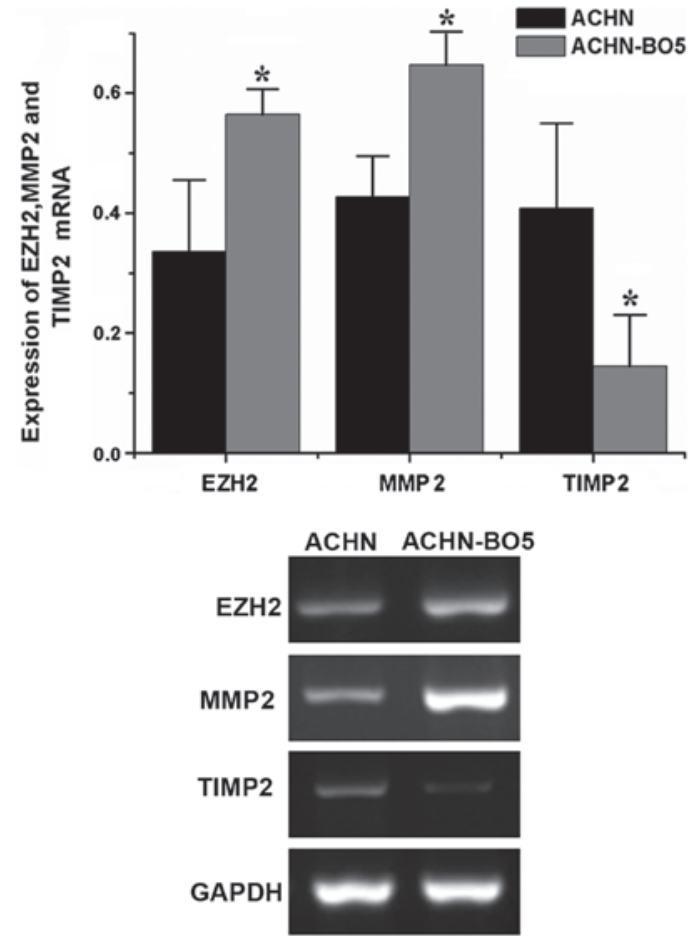

Figure 6. Expression of EZH2, MMP2 and TIMP2 mRNA in ACHN and ACHN-BO5 cell lines detected using semi-quantitative reverse transcription polymerase chain reaction. Values are presented as the mean \pm standard deviation $(\mathrm{n}=3)$. ${ }^{*} \mathrm{P}<0.05$ vs. ACHN cells. EZH2, enhancer of zeste homolog 2 MMP2, matrix metalloproteinase-2; TIMP2, tissue inhibitor of metalloproteinase-2; mRNA, messenger RNA.

performed in order to evaluate whether the altered expression of these three proteins occurred at the transcriptional level in the ACHN and ACHN-BO5 cell lines. The expression levels of EZH2 and MMP2 mRNA were higher in ACHN-BO5 cells than those in ACHN cells, whereas the expression levels of TIMP2 mRNA were lower in ACHN-BO5 cells than those in ACHN cells (Fig. 6).

TIMP2 is more highly methylated in ACHN-BO5 cells. The potential mechanism underlying the downregulated expression of TIMP2 mRNA in ACHN-BO5 cells was assessed using MSP. The TIMP2 promoter was more highly methylated in ACHN-BO5 cells than in ACHN cells (Fig. 7).

\section{Discussion}

Cancer metastasis is a complex process, where cancer cells migrate from their site of origin and invade other parts of the body via the bloodstream, lymphatic system or direct extension (19). Molecularly, tumor cells gain gene transcription capabilities and express various proteins and enzymes in order to degrade extracellular matrix proteins and invade adjacent tissues (20). However, the distant metastasis of various types of human cancer indicates preferences for certain organs; therefore, certain types of cancer tend to spread to particular organs and tissues (19). For example, breast cancer preferentially metastasizes to bone and lung tissue (21). RCC frequently metastasizes to the lungs, bone or brain, whereas the brain is most commonly the distant site of metastasis of melanoma (22).
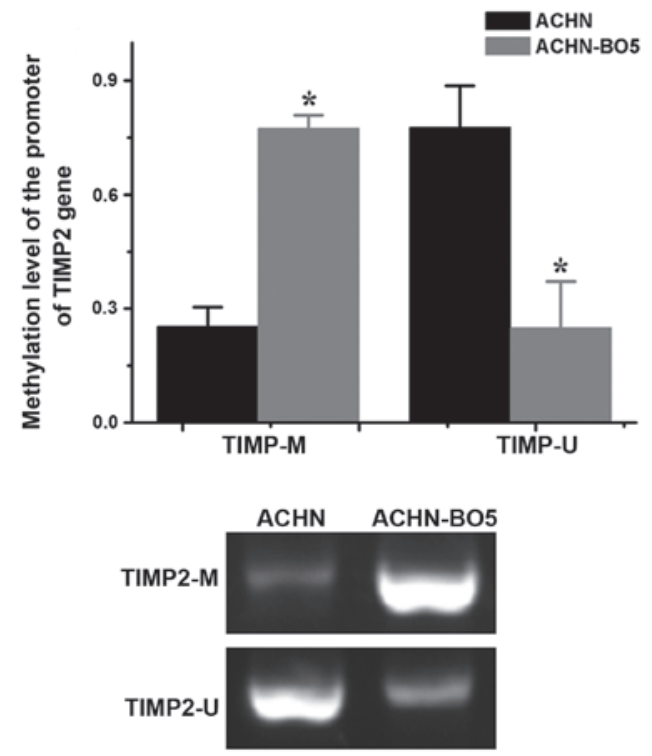

Figure 7. Methylation of the TIMP2 promoter assessed using methylation-specific PCR analysis. TIMP2-M is the PCR product obtained using a methylated primer and represents methylation of the TIMP 2 promoter, while TIMP2-U was produced using a demethylated primer and represents the demethylated TIMP2 promoter. Values are presented as the mean \pm standard deviation ( $\mathrm{n}=3$ ). ${ }^{*} \mathrm{P}<0.05$ vs. ACHN cells. TIMP2, tissue inhibitor of metalloproteinase-2; PCR, polymerase chain reaction.

Therefore, a study of organ-specific RCC metastasis may aid in the prevention of RCC progression. In a previous study by our group, an RCC cell line (ACHN-BO5) with high potential for metastasis to the bone was generated (9). These cells exhibited a significantly enhanced invasion and proliferation capacity in vitro, compared to that of the parental ACHN cells. The present study investigated whether cell adhesion molecules, including EZH2, MMP2 and TIMP2, contributed to alterations in the phenotype of tumor cells. Expression levels of EZH2 and MMP2 mRNA and protein were higher in ACHN$\mathrm{BO} 5$ cells than those in ACHN cells, whereas the expression of TIMP2 mRNA and protein was lower in ACHN-BO5 cells than that in ACHN cells. MSP data indicated that the downregulated expression of TIMP2 may be due to the methylation of the TIMP 2 promoter. Furthermore, the expression of these proteins was evaluated in tissue specimens from patients with RCC that had metastasized to the bone and patients with RCC without metastasis. The results confirmed those of the in vitro investigations, indicating that the expression of EZH2 and MMP2 protein was higher in tissues from patients with RCC that had metastasized to the bone than that in tissues from renal cancer patients without metastasis; and that there was no significant difference in the expression of TIMP2 protein between the two types of tissue.

The epigenetic modification enzyme, EZH2, has a homologous structure to the Drosophila E(z) gene, and was identified in the 1990s (23). Cardoso et al (24) demonstrated that the EZH2 protein was a key member of the polycomb group gene family and was able to modulate cell proliferation and the signaling pathway via the suvar3-9, enhancer of zeste, trtharax domain. The EZH2 protein is involved in mediating the development, metastasis, invasiveness and prognosis of various types of human cancer (23-32). Therefore, EZH2 is of 
interest in basic and clinical studies of cancer. Several studies have demonstrated that EZH2 protein is highly expressed in RCC tissues (33-36), the level of which is associated with $\mathrm{RCC}$ dedifferentiation, suggesting that the EZH2 protein may contribute to RCC progression and metastasis. More recently, studies have indicated that EZH2 protein is highly expressed in prostate cancer tissues, which are also associated with a high rate of metastasis to the bone $(16,37,38)$. Knockdown of EZH2 expression in metastatic bone tumors leads to atrophy of the metastatic bone foci and a reduction in or end to bone destruction (39), suggesting that EZH2 protein has a role in mediating metastasis to the bone.

MMPs, including MMP2, are required for degradation of the extracellular matrix and are specifically inhibited by the TIMPs. Therefore, these two families of proteins have significant roles in tumor metastasis. Numerous studies have confirmed that alterations in the balance of MMPs/TIMPs may lead to the metastasis of human cancers to the bone (40-43). Further studies have demonstrated that a downregulation of TIMP2 expression was associated with methylation of the TIMP2 promoter (44-50). Of note, EZH2 contains histone methyltransferase activity, which is able to silence genes via the methylation of $\mathrm{H} 3$ histone lysine 27 (51). It was therefore hypothesized that overexpression of $\mathrm{EZH} 2$ protein may lead to epigenetic silencing of TIMP2 expression. The results of the present study confirmed that there was an enhanced level of TIMP2 promoter methylation in ACHN-BO5 cells compared to that in ACHN cells. However, the results of the ex vivo investigations did not demonstrate an association between EZH2 expression and reduced TIMP2 protein, which does not support the hypothesis that EZH2 protein may be able to cause epigenetic silencing of TIMP2 expression. Due to the small sample size in the present study, further investigations are required in order to confirm this hypothesis.

Further studies are required to elucidate the molecular mechanisms underlying how the expression of EZH2, MMP2 and TIMP2 is altered in RCC tissues or cells with high potential for bone metastasis.

\section{Acknowledgements}

The present study was part of the programs on The Natural Science Foundation funded through The Science and Technology Department of Hubei Province, Wuhan, China (no. 2008CDB168) and The Science Foundation funded through The Health Department of Hubei Province, Wuhan, China (no. 2010JX5B03). This study was also part of the programs on The Nature Science Foundation funded by The Huazhong University of Science and Technology (2013QN200). The authors would like to thank all study participants and Medjaden Bioscience Limited (Hong Kong, China) for assisting in the preparation of this manuscript.

\section{References}

1. Kosary C and McLaughlin J: Kidney and renal pelvis. In: Cancer Statistics Review: 1973-1990. Miller BA, Ries LAG, Hankey BE, et al (eds). National Institutes Of Health, Bethesda, ppx1-x22, 1993.

2. Fleming $\mathrm{S}$ and Lewi $\mathrm{H}$ : Collecting duct carcinoma of the kidney. Histopathology 10: 1131-1141, 1986.
3. Thoenes W, Störkel S and Rumpelt H: Histopathology and classification of renal cell tumors (adenomas, oncocytomas and carcinomas). The basic cytological and histopathological elements and their use for diagnostics. Pathol Res Pract 181: 125-143, 1986.

4. Pisani P,Parkin D and Ferlay J: Estimates of the worldwide mortality from eighteen major cancers in 1985. Implications for prevention and projections of future burden. Int J Cancer 55: 891-903, 1993.

5. Ritchie A and Chisholm G: The natural history of renal carcinoma. Semin Oncol 10: 390-400, 1983.

6. Wood SL and Brown JE: Skeletal metastasis in renal cell carcinoma: current and future management options. Cancer Treat Rev 38: 284-291, 2012.

7. Rini BI, Campbell SC and Escudier B: Renal cell carcinoma. Lancet 373: 1119-1132, 2009.

8. Erdoğan F, Demirel A and Polat O: Prognostic significance of morphologic parameters in renal cell carcinoma. Int J Clin Pract 58: 333-336, 2004.

9. Wang J, Chen A, Yang C, Zeng H, Qi J and Guo FJ: A bone-seeking clone exhibits different biological properties from the ACHN parental human renal cell carcinoma in vivo and in vitro. Oncol Rep 27: 1104-1110, 2012.

10. Gibson WT, Hood RL, Zhan SH, et al; FORGE Canada Consortium: Mutations in EZH2 cause Weaver syndrome. Am J Hum Genet 90: 110-118, 2012.

11. Weaver DD, Graham CB, Thomas I and Smith DW: A new overgrowth syndrome with accelerated skeletal maturation, unusual facies, and camptodactyly. J Pediatr 84: 547-552, 1974.

12. Kleer CG, Cao Q, Varambally S, et al: EZH2 is a marker of aggressive breast cancer and promotes neoplastic transformation of breast epithelial cells. Proc Natl Acad Sci USA 100: 11606-11611, 2003.

13. Kehinde EO, Maghrebi M and Anim JT: The importance of determining the aggressiveness of prostate cancer using serum and tissue molecular markers. Can J Urol 15: 3967-3974, 2008.

14. Simon JA and Lange CA: Roles of the EZH2 histone methyltransferase in cancer epigenetics. Mutat Res 647: 21-29, 2008.

15. Varambally S, Cao Q, Mani RS, et al: Genomic loss of microRNA-101 leads to overexpression of histone methyltransferase EZH2 in cancer. Science 322: 1695-1699, 2008.

16. Shen L, Cui J, Liang S, Pang Y and Liu P: Update of research on the role of EZH2 in cancer progression. Onco Targets Ther 6: 321-324, 2013.

17. Nagase $\mathrm{H}$ and Woessner JF Jr: Matrix metalloproteinases. J Biol Chem 274: 21491-21494, 1999.

18. Shi SR, Key ME and Kalra KL: Antigen retrieval in formalinfixed, paraffin-embedded tissues: an enhancement method for immunohistochemical staining based on microwave oven heating of tissue sections. J Histochem Cytochem 39: 741-748, 1991.

19. Nguyen DX and Massagué J: Genetic determinants of cancer metastasis. Nat Rev Genet 8: 341-352, 2007.

20. Wong GS and Rustgi AK: Matricellular proteins: priming the tumour microenvironment for cancer development and metastasis. Br J Cancer 108: 755-761, 2013.

21. Drabsch Y and ten Dijke P: TGF- $\beta$ signaling in breast cancer cell invasion and bone metastasis. J Mammary Gland Biol Neoplasia 16: 97-108, 2011.

22. Zlotnik A, Burkhardt AM and Homey B: Homeostatic chemokine receptors and organ-specific metastasis. Nat Rev Immunol 11: 597-606, 2011.

23. Chen H, Rossier C and Antonarakis SE: Cloning of a human homolog of the Drosophila enhancer of zeste gene (EZH2) that maps to chromosome 21q22.2. Genomics 38: 30-37, 1996.

24. Cardoso C, Timsit S, Villard L, Khrestchatisky M, Fontès M and Colleaux L: Specific interaction between the XNP/ATR-X gene product and the SET domain of the human EZH2 protein. Hum Mol Genet 7: 679-684, 1998.

25. Heinen A, Tzekova N, Graffmann N, et al: Histone methyltransferase enhancer of zeste homolog 2 regulates Schwann cell differentiation. Glia 60: 1696-1708, 2012.

26. Xia H, Yu CH, Zhang Y, et al: EZH2 silencing with RNAi enhances irradiation-induced inhibition of human lung cancer growth in vitro and in vivo. Oncol Lett 4: 135-140, 2012.

27. Cho HM, Jeon HS, Lee SY, et al: microRNA-101 inhibits lung cancer invasion through the regulation of enhancer of zeste homolog 2. Exp Ther Med 2: 963-967, 2011.

28. Kikuchi J, Takashina T, Kinoshita I, et al: Epigenetic therapy with 3-deazaneplanocin A, an inhibitor of the histone methyltransferase EZH2, inhibits growth of non-small cell lung cancer cells. Lung Cancer 78: 138-143, 2012. 
29. Marchesi I, Fiorentino FP, Rizzolio F, Giordano A and Bagella L: The ablation of EZH2 uncovers its crucial role in rhabdomyosarcoma formation. Cell Cycle 11: 3828-3836, 2012.

30. Zhou J, Roh JW, Bandyopadhyay S, et al: Overexpression of enhancer of zeste homolog 2 (EZH2) and focal adhesion kinase (FAK) in high grade endometrial carcinoma. Gynecol Oncol 128: 344-348, 2013.

31. Wan L, Li X, Shen H and Bai X: Quantitative analysis of EZH2 expression and its correlations with lung cancer patients clinical pathological characteristics. Clin Transl Oncol 15: 132-138, 2013

32. Zhang R, Wang R, Chang H, et al: Downregulation of Ezh2 expression by RNA interference induces cell cycle arrest in the G0/G1 phase and apoptosis in U87 human glioma cells. Oncol Rep 28: 2278-2284, 2012.

33. Wagener N, Macher-Goeppinger S, Pritsch M, et al: Enhancer of zeste homolog 2 (EZH2) expression is an independent prognostic factor in renal cell carcinoma. BMC Cancer 10: $524,2010$.

34. Wagener N, Holland D, Bulkescher J, et al: The enhancer of zeste homolog 2 gene contributes to cell proliferation and apoptosis resistance in renal cell carcinoma cells. Int J Cancer 123: 1545-1550, 2008.

35. Hinz S, Weikert S, Magheli A, et al: Expression profile of the polycomb group protein enhancer of Zeste homologue 2 and its prognostic relevance in renal cell carcinoma. J Urol 182: 2920-2925, 2009

36. Sakurai T, Bilim VN, Ugolkov AV, et al: The enhancer of zeste homolog 2 (EZH2), a potential therapeutic target, is regulated by miR-101 in renal cancer cells. Biochem Biophys Res Commun 422: 607-614, 2012.

37. Li K, Chen MK, Situ J, et al: Role of co-expression of c-Myc, EZH2 and p27 in prognosis of prostate cancer patients after surgery. Chin Med J (Engl) 126: 82-87, 2013.

38. Yang YA and $\mathrm{Yu}$ J: EZH2, an epigenetic driver of prostate cancer. Protein Cell 4: 331-341, 2013.

39. Takeshita F, Minakuchi Y, Nagahara S, et al: Efficient delivery of small interfering RNA to bone-metastatic tumors by using atelocollagen in vivo. Proc Natl Acad Sci USA 102: 12177-12182, 2005.
40. Voorzanger-Rousselot N, Juillet F, Mareau E, Zimmermann J, Kalebic T and Garnero P: Association of 12 serum biochemical markers of angiogenesis, tumour invasion and bone turnover with bone metastases from breast cancer: a crossectional and longitudinal evaluation. Br J Cancer 95: 506-514, 2006.

41. Andela VB, Gordon AH, Zotalis G, et al: NFkappaB: a pivotal transcription factor in prostate cancer metastasis to bone. Clin Orthop Relat Res (415 Suppl): S75-S85, 2003.

42. Lhoták S, Elavathil LJ, Vukmirović-Popović S, Duivenvoorden WC, Tozer RG and Singh G: Immunolocalization of matrix metalloproteinases and their inhibitors in clinical specimens of bone metastasis from breast carcinoma. Clin Exp Metastasis 18: 463-470, 2000.

43. Yoneda T, Sasaki A, Dunstan C, et al: Inhibition of osteolytic bone metastasis of breast cancer by combined treatment with the bisphosphonate ibandronate and tissue inhibitor of the matrix metalloproteinase-2. J Clin Invest 99: 2509-2517, 1997.

44. Hsu CH, Peng KL, Kang ML, et al: TET1 suppresses cancer invasion by activating the tissue inhibitors of metalloproteinases. Cell Rep 2: 568-579, 2012.

45. Shin YJ and Kim JH: The role of EZH2 in the regulation of the activity of matrix metalloproteinases in prostate cancer cells. PloS One 7: e30393, 2012.

46. Fornari F, Milazzo M, Chieco P, et al: In hepatocellular carcinoma miR-519d is upregulated by p53 and DNA hypomethylation and targets CDKN1A/p21, PTEN, AKT3 and TIMP2. J Pathol 227: 275-285, 2012.

47. Matsusaka K, Kaneda A, Nagae G, et al: Classification of Epstein-Barr virus-positive gastric cancers by definition of DNA methylation epigenotypes. Cancer Res 71: 7187-7197, 2011.

48. Hervouet E, Vallette FM and Cartron PF: Dnmt3/transcription factor interactions as crucial players in targeted DNA methylation. Epigenetics 4: 487-499, 2009.

49. Martinez R, Schackert G and Esteller M: Hypermethylation of the proapoptotic gene TMS1/ASC: prognostic importance in glioblastoma multiforme. J Neurooncol 82: 133-139, 2007.

50. Chu LC, Eberhart CG, Grossman SA and Herman JG: Epigenetic silencing of multiple genes in primary CNS lymphoma. Int J Cancer 119: 2487-2491, 2006.

51. Crea F, Fornaro L, Bocci G, et al: EZH2 inhibition: targeting the crossroad of tumor invasion and angiogenesis. Cancer Metastasis Rev 31: 753-761, 2012. 